\title{
A Micro-Doppler Modulation of Spin Projectile on CW Radar
}

\author{
Zhi-Xue LIU \\ Baicheng Ordnance Test Center of China, Baicheng 137001, P. R. China
}

\begin{abstract}
To obtain the spin speed of projectile effectively, a micro-Doppler modulation model of rotating projectile measured by continuous-wave radar ( $\mathrm{CW}$ radar) is introduced. High spin speed of projectile brings micro-Doppler modulation on echoes of $\mathrm{CW}$ radar, and there are many micro-Doppler modulation harmonic waves in the zero intermediate frequency (ZIF) echoes. The frequency interval of the adjacent harmonic waves is several times of rotational frequency, but the integral multiple is unknown. The simulation results prove correctness of the proposed mathematic model.
\end{abstract}

\section{Introduction}

The spin speed is a key test parameter of motive projectile and directly related to the design of various projectiles, guidance devices and fuses etc. The main methods to measure projectile spin speed in early stage, such as telemetry method and optical method, have some defects [1]. To the telemetry method, some sensors must be added in the projectile, so it will change the structure of projectile. The optical method could only measure a little part of the ballistic trajectory and it is affected greatly by the environment.

In fact, high speed spin of projectile bring modulation on echoes of CW radar, and there are many harmonic waves around Doppler signal, which called micro-Doppler effect [2]. In recent years, spin speed is measured by continuous wave Doppler radar, through grooving the bottom of the projectile, which can strengthen the micro-Doppler signal [3]. Compared with optical method, this method can measure the spin speed of projectile along with time, but it also changes the structure of projectile as the telemetry method. In order to measure spin speed with unbroken projectile, a method based on digital demodulation is proposed by extracting the micro-Doppler signal in the echo of $\mathrm{CW}$ radar, which is the first application of micro-Doppler technology on unbroken projectile [4]. But the mathematical model proposed by References 4 is incomplete. The method proposed in References 4 is incomplete, and the extracted spin speed is ambiguous. So, an emendatory micro-Doppler modulation of rotating projectile on $\mathrm{CW}$ radar echoes is proposed, which explains the reason of spin speed ambiguous.

\section{Modulation of rotating projectile}

The structure of uncontrolled projectile, such as cannonballs, rockets and etc, is symmetrical. Without loss of generality, it is supposed that there are $N$ scattering pointers $p_{1}, \cdots, p_{i}, \cdots, p_{N}$, evenly

\footnotetext{
${ }^{a}$ Corresponding author: 1iuzx09@qq.com
} 
distributed on the bottom edge, which are $l$ away from the center of the bottom shown as Figure 1. In the coordinate system of projectile axis, the coordinates of $p_{i}$ is $X_{i}=\left(0, l \cdot \sin \theta_{i}, l \cdot \cos \theta_{i}\right)^{T}$, where $\theta_{i}=2 \pi f_{r} t+2 \pi i / N, f_{r}$ is the spin frequency of projectile.

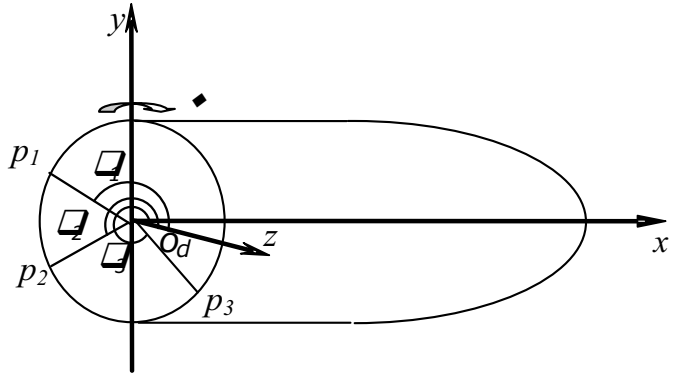

Figure 1. Rotation diagram of projectile

Radar measurement coordinate system $O_{r} X Y Z$ and reference coordinate system $O_{d} X_{N} Y_{N} Z_{N}$ is established shown as Figure 2. The origin of radar measurement coordinate system is the radar, and the $O_{r} X$ axis parallels the firing azimuth of the gun. The origin of reference coordinate is the center of the projectile bottom, and the $O_{d} X_{N}$ axis parallels the direction of velocity. Reference coordinate system moves with the projectile. In the process of launching, the $O_{r} X$ axis of radar measurement coordinate system almost parallels the direction of velocity. So the angular altitude $\beta$ of velocity in Figure 2 is equal to the elevation $E_{l}$ of radar in measurement coordinate system, and the azimuth $\psi$ of velocity is equal to azimuth $A_{z}$ of radar. Usually, radar is placed close to the firing azimuth of the gun, so the measuring azimuth $A_{z}$ of radar is very small, and so it also can be considered $A_{z}=0$ here. After twice rotations, the coordinates of $p_{i}$ in reference coordinate system can be transformed into the coordinates of $p_{i}$ in the coordinate system of projectile axis. First, reference coordinate system rotates around the new $\mathrm{Y}$ axis with $\psi$, and then rotates around the $\mathrm{Z}$ axis with $\beta$. The $\beta$ is elevation of the projectile axis and $\psi$ is azimuth of the projectile axis. Therefore the coordinates of scattering pointer $p_{i}$ in reference coordinates is shown as Eq. 1.

$$
\left[\begin{array}{l}
x_{i} \\
y_{i} \\
z_{i}
\end{array}\right]=\left[\begin{array}{ccc}
\cos E_{l} & -\sin E_{l} & 0 \\
\sin E_{l} & \cos E_{l} & 0 \\
0 & 0 & 1
\end{array}\right] \cdot X_{i}
$$

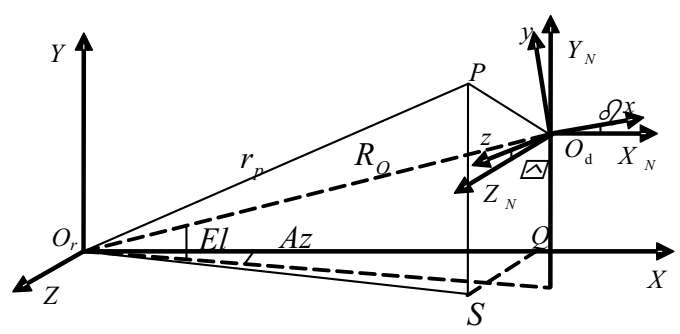

Figure 2. The relationship of coordinate systems

The slant range $r_{i}$ between $p_{i}$ and radar is shown as Eq. 2 . 


$$
\begin{aligned}
r_{i}= & {\left[\left(R \cos E_{l} \cos A_{z}-l \cdot \sin E_{l} \cdot \sin \theta_{i}\right)^{2}\right.} \\
& +\left(R \cos E_{l} \sin A_{z}+l \cdot \cos E_{l} \cdot \sin \theta_{i}\right)^{2} \\
& \left.+\left(R \sin E_{l}+l \cdot \cos \theta_{i}\right)^{2}\right]^{1 / 2} \\
& =\left[R^{2}+2 R\left(a \cdot \cos \theta_{i}+b \cdot \sin \theta_{i}\right)+l^{2}\right]^{1 / 2}
\end{aligned}
$$

Where, $a=l \sin E_{l} ; b=l \cos E_{l} \sin \left(A_{z}+E_{l}\right)$. Within a certain time, azimuth $A_{z}$, elevation $E_{l}$ and $\beta$ vary slowly compared with micro-motion, so they can be considered as constant. Because $R>>l, r_{i}$ can be approximated as Eq. 3 based on Taylor expansion.

$$
\begin{aligned}
r_{i} & \approx R+\left(a \cdot \cos \theta_{i}+b \cdot \sin \theta_{i}\right) / 2 \\
& =R+d \cos \left(\theta_{i}+\xi\right)
\end{aligned}
$$

Where, $d=\left(a^{2}+b^{2}\right)^{1 / 2} / 2 ; \xi=\operatorname{ctg} E_{l} \cdot \sin \left(A_{z}+E_{l}\right)$.

The continuous wave radar transmits a single tone shown as Eq. 4 .

$$
s_{T}(t)=\cos (2 \pi f t)
$$

In Eq. $4, f$ is the frequency of single tone. While the single tone illuminates the rotating projectile, the echo from the scattering pointer $p_{i}$ is shown as Eq. 5 .

$$
s_{i}(t)=m_{i 0} \cdot \cos \left[2 \pi f\left(t-2 r_{i} / c\right)\right]
$$

In Eq. $5, m_{i 0}$ is the amplitude of the echo from the scattering pointer $p_{i}$. Put Eq.3 into Eq.5, then

$$
s_{i}(t)=m_{i 0} \cdot \cos \left\{2 \pi f t-4 \pi f R / c+\Delta \varphi_{i}\right\}
$$

Where $2 \pi f t$ is the phase varying with time of single tone, $4 \pi f t R / c$ is the phase retardation caused by the range between the radar and the projectile, $\Delta \varphi_{i}$ is the phase shift caused by rotation of projectile and shown as Eq.7.

$$
\begin{aligned}
\Delta \varphi_{i} & =-4 \pi f d \cos \left(\theta_{i}+\xi\right) / c \\
& =-4 \pi f d \cos \left(2 \pi f_{r} t+2 \pi i / N+\xi\right) / c
\end{aligned}
$$

In Eq. 7, the form of $\Delta \varphi_{i}$ is same with the phase of frequency modulation, which means that the phase shift is the frequency modulation [5]. According to the frequency modulation theory, while $\left|\Delta \varphi_{i}\right|>\pi / 6$, the echo from the scattering pointer $p_{i}$ can be expanded to a trigonometric series shown as Eq. 8, which coefficients are Bessel function.

$$
\begin{aligned}
s_{i}(t) & =\sum_{k=-\infty}^{+\infty} m_{i 0} J_{k}\left(\beta_{F M}\right) \cos (2 \pi f t \\
& \left.-4 \pi f R / c+2 \pi k f_{r} t+2 \pi i k / N+k \xi\right)
\end{aligned}
$$

In Eq. $8, J_{k}\left(\beta_{F M}\right)$ is the $k$ th Bessel function, $\beta_{F M}=4 \pi f d / c f_{r}$ is the frequency modulation coefficient. The ZIF echoes are shown as Eq. 9. 


$$
\begin{aligned}
x_{i}(t) & =m_{i} \sum_{k=-\infty}^{+\infty} J_{k}\left(\beta_{F M}\right) \\
& \cdot \exp \left[j 2 \pi\left(f_{d}+k f_{r}\right) t+2 \pi k i / N\right]
\end{aligned}
$$

In Eq. $9, m_{i}$ is the amplitude of the ZIF echoes from $p_{i}, f_{d}$ the is the Doppler frequency. The ZIF echoes from all scattering pointers are shown as Eq. 10.

$$
x(t)=\sum_{k=-\infty}^{\infty} M_{k} \exp \left[j 2 \pi\left(f_{d}+k f_{r}\right) t\right]
$$

In Eq. $10, M_{k}$ is the amplitude of micro-Doppler modulation harmonic waves and shown in Eq. 10.

$$
M_{k}=J_{k}\left(\beta_{F M}\right) \sum_{i=1}^{N} m_{i} \exp (j 2 \pi k i / N)
$$

While the scattering are equal, the amplitude of echoes from different scattering points are equal, then $m_{1}=m_{2}=\cdots=m_{N}$. The amplitude of harmonic wave, which frequency is $k f_{r}$, is shown in Eq.11.

$$
M_{k}=\left\{\begin{array}{cc}
N J_{k}\left(\beta_{F M}\right) m_{1} & k=N K \\
0 & k \neq N K
\end{array}\right.
$$

In Eq.11, $K$ is an integer, and the harmonic wave which frequency is not $N f_{r}$ disappeared.

The mathematic model in Eq. 10 shows that the ZIF echoes from the rotating projectile contain the micro-Doppler modulation harmonic wave. The frequency interval of the adjacent harmonic waves is the rotational frequency, and the frequency interval between the adjacent harmonic waves to the Doppler signal is also several times of the rotational frequency. In general, all harmonics should exist as the Eq. 11 shows, but in some case some harmonic are disappeared as the Eq. 12 shows. Compared Eq. 11 and Eq. 12, the frequency interval of the adjacent harmonic wave is indefinite in different conditions, and the spin frequency cannot be extracted directly from the frequency interval between the adjacent harmonic waves or the harmonic frequency.

\section{Processing of micro-doppler signal}

In the ZIF echoes from the rotating projectile, the micro-Doppler modulation harmonic wave is weaker than the Doppler signal, and the frequency interval between the harmonic waves and the Doppler signal is also very small, so the micro-Doppler modulation harmonic wave are submerged by noise in the power spectrum calculated by the Fourier transform. In the practical engineering, zoomFFT is used for analyzing the spectrum of micro-Doppler modulation harmonic wave at high resolution .

After the Doppler frequency $f_{d}$ is obtained, a local oscillator can be structured as Eq. 13.

$$
S_{1}(t)=e^{-j 2 \pi f_{d} t}
$$

Using $s_{1}(t)$, then the ZIF echoes $x(t)$ can be demodulated to a new signal shown in Eq. 14 .

$$
\begin{aligned}
x_{r}(t) & =x(t) s_{1}(t)=x(t) e^{-j 2 \pi f_{d} t} \\
& =\sum_{k=-\infty}^{\infty} M_{k} \exp \left[j 2 \pi\left(k f_{r}\right) t\right]
\end{aligned}
$$


In the new signal $x_{r}(t)$, the Doppler signal is shifted to zero frequency, and the micro-Doppler modulation harmonic wave, which frequency is $f_{d+} k f_{r}$, are shifted to $k f_{r}$. Then the new signal $x_{r}(t)$; can be named as the micro-Doppler signal.

To improve the spectrum resolution and restrain the frequency aliasing, zoom-FFT adopts a low rate re-sampling and a low pass filter. Another Fourier transform can calculate the spectrum of $x_{r}(t)$, and the frequency peak in spectrum is the frequency of micro-Doppler modulation harmonic wave.

\section{Experiments}

In this section, the correctness of the proposed mathematic model is proved through two simulation experiment. The control parameters used in our simulation are summarized in Table 1.

Table 1. Parameters used in the simulation example.

\begin{tabular}{|c|c|}
\hline Center frequency & $10 \times 10^{10} \mathrm{~Hz}$ \\
\hline Sample interval & $20 \mu s$ \\
\hline Radial velocity & $640-9.97 t \mathrm{~m} / \mathrm{s}$ \\
\hline Spin speed & $100-1.2 t+0.05 * t^{2} \mathrm{~Hz}$ \\
\hline Radial range & Eq. 2 \\
\hline Scattering point & 2 or 4 \\
\hline
\end{tabular}

In the first experiment, the amplitude of the echo from scattering pointer is a random number that obeys the $(1,0.5)$ normal distribution. The spectrogram and power spectral density are shown in Figure 3. In Figure 3 (a), the spectrogram of the micro-Doppler signal from the rotating projectile with 2 scatter is shown in Fig. 3 (a). Fig. 3 (b) shows the power spectrum curve at the first time point in Figure 3 (a). In Figure 3 (c), the spectrogram of the micro-Doppler signal from the rotating projectile with 4 scatter is shown. Figure 3 (d) shows the power spectrum curve at the first time point. It is shown from Figure 3 that, the frequency interval of the adjacent harmonic waves in the micro-Doppler signal from scatters with random scattering coefficient is the rotational frequency. The frequency interval of the adjacent harmonic waves is several times of the rotational frequency, but the integral multiple is unknown.

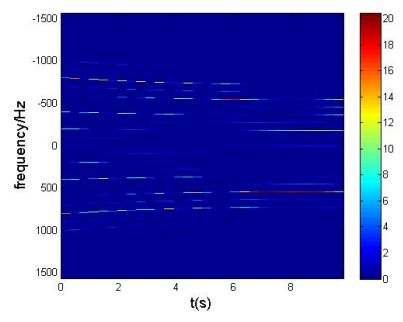

(a) spectrogram of 2 scatter

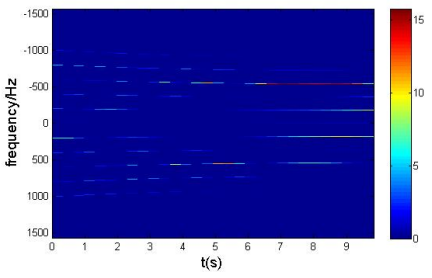

(c) spectrogram of 4 scatter

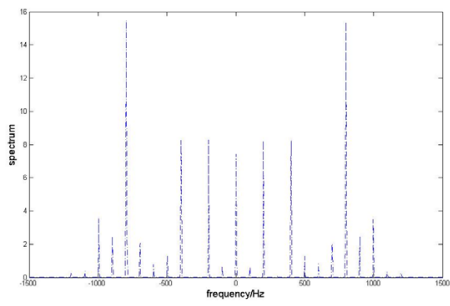

(b) spectrum on $1^{\text {st }}$ point of 2 scatter

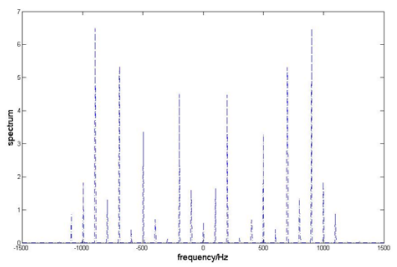

(d) spectrum on $1^{\text {st }}$ point of 4 scatter

Figure 3. The Spectrogram and spectrum of random scattering 
In the second experiment, the amplitude of the echo from scattering pointer is equal instead of the random distribution. The results are shown in Figure 4.

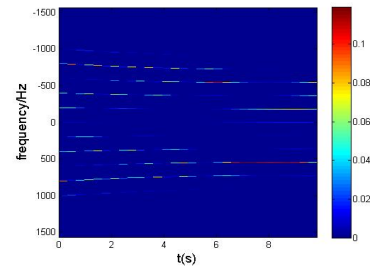

(a) spectrogram of 2 scatter

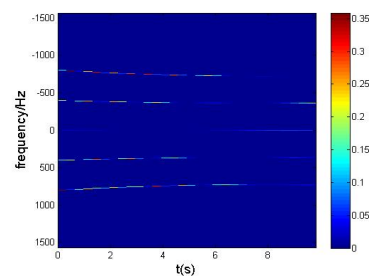

(c) spectrogram of 4 scatter

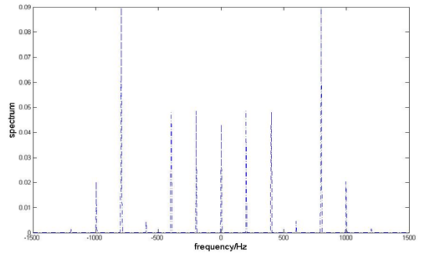

(b) spectrum on $1^{\text {st }}$ point of 2 scatter

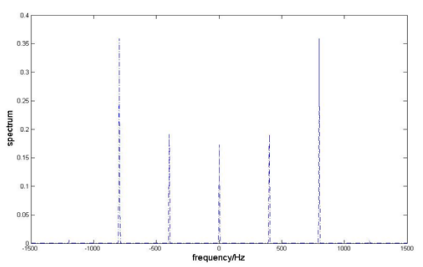

(d) spectrum on $1^{\text {st }}$ point of 4 scatter

Figure 4. The spectrogram and spectrum of equal scattering

It is shown from Figure 4 that, the frequency interval of the adjacent harmonic waves in the microDoppler signal from scatters with identical scattering coefficient is several rotational frequencies. The frequency interval of the adjacent harmonic waves is the integer times of rotational frequency, and the integral multiple is the number of scatters.

Compare the result of Fig. 3 and Fig. 4, the frequency interval of the adjacent harmonic waves is indefinite in different conditions, it cannot be extracted directly from the radar echoes.

\section{Conclusions}

The micro-Doppler modulation model of the projectile rotation with multi-scatters is established based on the relationship between the ZIF echoes of projectile measured by CW radar and projectile rotation. It is deduced that the frequency interval of the adjacent harmonic waves in the micro-Doppler signal is several times of the spin speed of projectile, and the times of the frequency interval to spin speed cannot be processed directly from the micro-Doppler signal. In the practical, the multiple between the frequency interval and the spin speed can be calculated with the help of other equipment. From the results of simulation, it is concluded that the micro-Doppler modulation model proposed in the paper is correct.

\section{References}

1. Han Zipeng. Projectile and Rocket Exterior Ballistics [M]. Beijing: Beijing University of Aeronautics and Astronauts press, 2008, 153-162.

2. V C Chen, F Li,S Ho,et al. Micro-Doppler effect in radar-phenomenon, model and simulation study [J]. IEEE Transactions on Aerospace and Electronic Systems, 2006, 42(1):2-21.

3. Wang Yuan-qin et al. A robust RCS periodicity estimation algorithm for ballistic target [J]. Aerospace Electronic Warfare, 2008, 24(2): 5-8.

4. Zhang Wan-jun, Wu Xiao-ying, Leng Xue-bing, et al. Testing Method of Projectile Rotating Speed Based on Micro-Doppler Effect of CW Radars [J]. Journal of Academy of Armored Force Engineering. 2012, 26(5):47-50.

5. Proakis, J. G., Digital Communications, 3rd ed., New York, McGraw-Hill, 1995. 